no occasion for apprehension. We are not the sole occupiers of the world, however, and I am informed that every time an atom bomb or hydrogen bomb is exploded the radioactivity of natural waters is raised inevitably throughout the world-the effect is by no means a localized one. The consequences remain to be seen, and I hazard no guess as to what they are likely to be.

Superficial though my remarks have been, I hope I may have succeeded in conveying the impression that, in the modern state, the problem of water supply is a general one. Man's personal need cannot be disassociated from the need of industry and these have to be made compatible. Conditions are such that water must be reused, often many times, if it is to be available in sufficient quantity. The avoidance of unnecessary pollution from both domestic and industrial sources should be the aim of every one of us in helping those whose business it is to ensure that the quality is of the required standard.

REFERENCES

Kenny, A. W. (1956). Bull. World Hlth Org. 14, 1007.

Ministry of Agriculture and Fisheries (1954). Tech. Bull. Minist. Agric., Lond., no. 4.

Ministry of Housing and Local Government (1956). Report of the Committee on Synthetic Detergents. London: H.M. Stationery Office.

\title{
Functions of water in the body
}

By J. R. RobInson, Medical Research Council Department of Experimental Medicine, University of Cambridge

Water, as Henderson (I9I3) pointed out, was uniquely suited to become the inorganic basis of living matter. Its unusually high dielectric constant helps to bring ions into solution in the lower part of the range of temperatures in which it is liquid, and dissolved ions play a large part in maintaining essential properties of cell membranes and the activity of enzymes. Ions in solution are, moreover, surrounded by mantles of electrostatically bound water molecules which modify their properties in biologically important ways. The great solvent power of water has leached from primeval rocks the elements we now find accumulated in living organisms; and life, as we know it, is only possible between the temperatures at which proteins are denatured and dilute solutions freeze.

Hydrolysing enzymes which catalyse reactions into which water enters as a reactant are mostly found outside cells, reducing foodstuffs to a suitable molecular size for absorption. Within cells, water appears rather as a physical substratum than as a chemical participant in the essential reactions which maintain life. Its other great function in the body is to provide a vehicle for the transport of raw materials, waste products, and the heat liberated by chemical action.

When we say that water makes up two-thirds of our bodies, we do not mean that the body is simply an odd-shaped bag two-thirds full of water. If this were so, the 
volume of water could be deduced from the concentration attained after a known amount of any solute had been dissolved in it and evenly mixed. All solutes should lead to the same volume; but in the body different solutes give different answers. Some, like urea, antipyrine, deuterium and tritium oxides, indicate a volume which must be that of all the water in the body; it agrees with the amount found by drying after death. Others, like colloidal dyes and labelled albumin, give a volume which is as clearly that of the blood plasma. Yet others give rather consistently an intermediate figure of about one-third of the total body water. These last are substances like inulin, mannitol, sucrose, and ions to which cell membranes in general are impermeable. The fraction which they delimit has been crudely identified with the extracellular water of the body-which forms an extremely tortuous continuum lying between and around the cells, in the blood and lymphatic vessels, and in the serous cavities. The intracellular fluids, by contrast, do not form one continuous fluid mass, but a multitude of tiny droplets isolated by the restricted permeability of the cell membranes. Hence all exchanges between one cell and another, or between a cell and the external environment, must take place through the extracellular fluids, which have been aptly called the 'middleman fluids' of the body.

The body water is not, of course, fresh water, but contains salts in solution; and the salts inside and outside the cells are characteristically different. The chief cation of the extracellular fluid is sodium; there are also small but important concentrations of potassium and calcium, and chloride and bicarbonate make up the bulk of the accompanying anions. The chief cation in the cells is potassium and the accompanying anions are probably mostly organic phosphates and proteins. Since some cells contain considerably more sodium and chloride than those of muscle and nerve, which have been studied most completely and are usually regarded as typical, there are really many intracellular fluids but only one extracellular fluid exhibiting no more than minor regional differences in composition.

And so, though now we live in air on dry land, our cells still lead an aquatic existence, as most cells must. Yet they are not quite like amoebas in a pond. Ponds are usually cold, and huge compared with the volume of amoebas in them, so that metabolic reactions are slow and do not sensibly alter the composition of the pondwater, which provides an unlimited source of food and an unlimited sink for the disposal of waste products. The water in our pond is more like warm sea-water than cold fresh water. Metabolism runs faster in warmer cells; and besides, many of ours have become highly specialized, and at the same time intolerant of changes in external composition or temperature. Moreover, our pond, so far from being vast compared with the cells inhabiting it, has only about one-third of their total volume, so that the activity of the cells does affect the composition of the pond, and would do so far more but for the regulating mechanisms which have been developed to prevent this. Specialization has made it at once more necessary and more difficult to 'fix' the milieu interieur; and so a great part of physiology is concerned with mechanisms which enable the little overcrowded pond under our skins to maintain our cells as though it had an unlimited volume. Our cells, living in their common pond, can send messages to each other by adding substances to the fluid around 
them. These may be special products of few cells which influence many, like thyroxine, or general products of many cells which specially affect few, like carbon dioxide. For the purpose of humoral co-ordination it is an advantage to keep the pond small; chemical messages spread faster through a smaller pond because smaller changes in quantity lead to larger changes in concentration, and it is mainly to concentrations that the brisker regulating mechanisms respond.

The chief disadvantages of the smallness of our pond arise from the paradox that several important functions of water in the body can only be fulfilled by losing it. From 0.5 to 1.51 , according to the concentrating power of the kidneys, must be lost every day as a vehicle for dissolved substances excreted in the urine. Far more water than this may have to be expended to control the body's temperature. Although the high specific heat of water provides an immediate thermal buffer which is the first line of defence against damaging local increases in the temperature of active tissues, and distribution of heat by the circulation provides a second line, the actual removal of heat from the body often depends upon the evaporation of sweat. Evaporation is the only means of cooling in an environment above body temperature, and temperature control takes precedence over the conservation of water. In hot places more than ro l. a day may be lost; and, if it were not for the high latent heat of vaporization of water, the loss would have to be proportionately greater.

Apart from sweating, water is unavoidably lost by evaporation from the moist outer layers of the skin and from the lungs and air passages. These losses may amount to no more than $\frac{2}{3} 1 . /$ day under ordinary conditions at sea level. At high altitudes where the atmospheric pressure is low, a lung-full of air brings in less oxygen than at sea level, but, since the vapour pressure of water is determined by the body temperature, it still takes away as much water when we breathe out. The increased pulmonary ventilation needed to supply oxygen to the muscles of a climber high on a mountain leads to enormous losses of water--and of heat, since the water is lost as vapour-in the expired air.

Our main concern from a nutritional point of view is with how these inevitable losses are made good; for water can only be an efficient vehicle of transport in the body if the circulation can promote thorough mixing and eliminate local differences in composition and temperature. The adequacy of the circulation depends upon the volume of circulating blood, which tends to follow changes in the volume of the extracellular fluid as a whole. The volume of extracellular fluid in its turn depends upon the balance of exchanges both with the cells and with the outer world. It will be convenient to deal first with exchanges of water within the body between the cells and the extracellular fluid.

Cell membranes are generally permeable to water, and in the absence of 'water pumps' (in which it is not fashionable to believe) water moves passively by diffusion from regions where its concentration is higher to regions where it is lower. This net movement occurs simply because diffusion is a random process. Since molecules in thermal motion are equally likely to jump in all directions, the number moving in any specified direction is proportional to the number present; more leave regions 
where there are many than regions where there are fewer. A semi-permeable membrane allows the net movement of water to continue but stops the diffusion of solutes. Hence water moves across such a membrane towards the higher concentration of solute. This net movement of water is called osmosis, and it is important to note that the movement of water is not due to osmotic pressure. Osmotic pressure is not the cause of osmosis, but the hydrostatic pressure that would be required to stop it.

The volume of a cell in equilibrium with a constant external osmotic pressure will be proportional to the quantity of solutes inside it; and this is profoundly influenced by metabolic activity. Interruption of respiration can increase the amount of intracellular solutes by permitting autolytic breakdown of large molecules into more numerous smaller ones, and by halting the mechanism which normally expels sodium. Although the increase from these causes is partly offset by loss of potassium, cells might be expected to swell if they were chilled, deprived of oxygen or poisoned with cyanide, or if essential metabolic sequences were disorganized, as by dinitrophenols. It has been clearly established that cells do swell in this way, but it is not yet quite certain how far interference with active transport of water may also contribute to the swelling (Robinson, I954, I956). The semipermeable membranes round cells are in general too tenuous to withstand appreciable differences in hydrostatic pressure, yet differences in concentration whose equivalents in osmotic pressure run into many atmospheres are encountered in living systems, so that some cells do appear capable of moving water in defiance of osmotic gradients. This property may be restricted to cells of certain secreting organs, such as the sweat and salivary glands and the kidneys. These cells line frontiers between the body and what is outside it, and some of them expend metabolic energy to shield other cells deeper within the body from external osmotic variations. But even though the influence of metabolic activity upon exchanges of water confers a certain independence upon individual cells, so that they do not respond to changes in extracellular osmotic pressure precisely like dead osmometers, yet so long as metabolism is proceeding normally, the osmotic pressure of the extracellular fluid is the most important factor in controlling the net exchange of water between the cells and the extracellular fluid.

The osmotic pressure of the extracellular fluid is kept remarkably constant in health-perhaps more so than any other physiological quantity-by the co-operation of two regulating mechanisms. As soon as the osmotic pressure of the body fluids begins to rise, we are prompted by thirst to ingest water, and an antidiuretic hormone is released from the neurohypophysis which delays the loss of water through the kidneys by promoting tubular reabsorption. If, conversely, the extracellular osmotic pressure begins to fall, the release of this hormone is suppressed, and there ensues a temporary and physiological state of diabetes insipidus during which excess water is quickly eliminated in a copious flow of dilute urine (Verney, 194I).

'Insensible' losses from the lungs and skin are of pure water, and so they raise the osmotic pressure of the extracellular fluid and evoke thirst to restore as much water as has been lost. Salt is lost as well as water in sweat, but the sweat is hypotonic so 
that sweating also increases the concentration of the extracellular fluid and evokes thirst. If, however, only sufficient water is ingested to correct the osmotic pressure, the volume of the extracellular fluid will not be restored to normal; and if, instead, the volume of water lost by sweating is replaced without the salt, the extracellular fluid will be diluted, and movement of water into cells by osmosis may cause such manifestations of water intoxication as stoker's or miner's cramp. Water intoxication may be produced experimentally by ingesting large amounts of water whilst diuresis is prevented by pituitary extracts. It may also occur if too much water without sodium chloride is given to patients who are releasing excessive amounts of antidiuretic hormone after surgical operations, or who have too little functioning kidney tissue to respond to the absence of the hormone from the circulating blood. It has been seen in anuric patients who have broken down their own tissues and flooded the extracellular compartments with metabolic water and water from the cells. There may be no dramatic symptoms when the condition arises insidiously in ways like these, though it may have fatal consequences by reducing the patient's capacity to withstand his illness (Hamburger \& Mathé, I 954; Wynn \& Rob, I954). Hormonally controlled water diuresis is probably more important to man than to other animals, for men are well known to drink for reasons and in quantities which bear scant relation to their physiological needs for water, and rely on this diuresis to protect them from the hazards of water intoxication.

Finally, since the osmotic pressure of the extracellular fluid is, other things being equal, the chief upholder of the balance between intra- and extra-cellular water, we can look upon thirst and water diuresis in a new light. They are not merely the means of keeping extracellular osmotic pressure constant, but the mechanisms which regulate the volume of the intracellular fluids. Besides thus controlling the volume of the cells, they set the stage for adjusting the volume as well as the concentration of the extracellular fluid; for about $90 \%$ of the osmotic pressure of the extracellular fluid is contributed by sodium and the anions accompanying it. It follows that, so long as the total concentration is kept constant, the volume of the extracellular fluid must be, to a first approximation, proportional to the amount of sodium in it. And, so, since the intake of sodium usually exceeds its loss by extrarenal channels, the kidneys are able to regulate the volume of extracellular fluid by controlling the excretion of sodium in the urine. They thus control the volume of water inside the cells by excreting water, and the volume of water outside the cells by excreting salt.

\section{REFERENCES}

Hamburger, J. \& Mathé, G. (1954). In Ciba Foundation Symposium on The Kidney, p. 288. [A. A. G. Lewis and G. E. W. Wolstenholme, editors.] London: J. \& A. Churchill.

Henderson, L. J. (I913). The Fitness of the Environment. New York: The Macmillan Co.

Robinson, J. R. (1954). Symp. Soc. exp. Biol. no. 8, p. 42.

Robinson, J. R. (1956). F. Physiol. 134, 216.

Verney, E. B. (194I). Proc. roy. Soc. B, 135, 25.

Wynn, V. \& Rob, C. G. (I954). Lancet, 266, 587. 